\title{
Mesoscopic self-assembly: a shift to complexity
}

\author{
Massimo Mastrangeli * \\ Physical Intelligence Department, Max Planck Institute for Intelligent Systems, Stuttgart, Germany
}

By focusing on the construction of thermodynamically stable structures, the self-assembly of mesoscopic systems has proven capable of formidable achievements in the bottomup engineering of micro- and nanosystems. Yet, inspired by an analogous evolution in supramolecular chemistry, synthetic mesoscopic self-assembly may have a lot more ahead, within reach of a shift toward fully three-dimensional architectures, collective interactions of building blocks and kinetic control. All over these challenging fronts, complexity holds the key.

Keywords: complexity, kinetics, mesoscopic systems, programmable matter, self-assembly

\section{OPEN ACCESS}

Edited by:

Andreas Dietzel,

Technische Universität Braunschweig,

Germany

Reviewed by:

Hamid Reza Barzegar,

Umeå University, Sweden

Christian Bergaud,

Centre national de la recherche

scientifique, France

*Correspondence:

Massimo Mastrangeli,

Physical Intelligence Department,

Max Planck Institute for Intelligent

Systems, Heisenbergstr. 3, Stuttgart

70569, Germany

mastrangeli@is.mpg.de

Specialty section: This article was submitted to Microand Nanoelectromechanical Systems, a section of the journal Frontiers in Mechanical Engineering

Received: 20 April 2015

Accepted: 01 June 2015

Published: 16 June 2015

Citation:

Mastrangeli M (2015) Mesoscopic self-assembly: a shift to complexity.

Front. Mech. Eng. 1:6.

doi: 10.3389/fmech.2015.00006

\section{Self-Assembly Everywhere, Particularly in the Middle}

Self-assembly is a powerful constructional heuristic rooted in concepts and methods of supramolecular chemistry (Whitesides et al., 1991; Mattia and Otto, 2015), and ultimately inspired by living matter (Nicolis and Prigogine, 1977) and unsupervised pattern formation (Ball, 2011). Over the last three decades, the synthesis of artificial systems by self-assembly has witnessed growing success and expanding technological reach (Groß and Dorigo, 2008; Grzybowski et al., 2009; Mastrangeli et al., 2009; Tørring et al., 2011; Mattia and Otto, 2015). Self-assembly has become synonymous of bottom-up systems engineering, complementing, or competing with more established topdown strategies. Under proper conditions, self-assembly may, in principle, be adapted to work at arbitrary physical scales (Whitesides and Grzybowski, 2002). Each scale nonetheless requires specifically tailored implementations of the general heuristic. Scale selectivity is dictated by the scaledependent hierarchy of forces that provide the interactions among building blocks, and by the sizedependent options available to efficiently fabricate the blocks themselves (from chemical synthesis to $3 \mathrm{D}$ printing, from micro/nanofabrication to numerically controlled machining). The conjuncture of fundamental laws and manufacturing opportunities does provide constraints on the types of embodiments associated with each physical domain (Whitesides and Grzybowski, 2002). However, it does not univocally prescribe possibilities, as documented by the mounting variety of ingenious implementations reported in literature (Grzybowski et al., 2009; Mastrangeli et al., 2009; Tørring et al., 2011; Crane et al., 2013). In between such continuum of physical scales and building block sizes lies a peculiar domain, whereby the objects to be assembled are too small to be efficiently handled by robotic effectors, and at the same time, too big to be addressed through supramolecular chemistry and DNA-based self-assembly (Tørring et al., 2011). This mesoscopic domain has fuzzy boundaries, which shrink with technological development, and currently concerns pre-existing building blocks of a few to a few hundreds micron in characteristic size (Mastrangeli et al., 2009; Leong et al., 2010; Crane et al., 2013). Several non-covalent interactions (e.g., capillary, electric, magnetic, hydrodynamic, hydrophobic, steric, depletive) (Bishop et al., 2009; Israelachvili, 2011) available in this domain can be tuned to a certain extent, and are not overwritten by gravity. Still, inertial effects overshadow the Brownian motion of mesoscopic building blocks - with the remarkable exception of active or self-propelling particles (Vicsek and Zafeiris, 2012; Marchetti et al., 2013). These traits 
render the mesoscopic domain singularly challenging for the implementation and fundamental understanding of self-assembly. Its fascinating features provide opportunities that continue to draw significant and multidisciplinary research efforts (Whitesides and Boncheva, 2002). Conversely, originally dedicated solutions have been exported to collateral fields (Whitesides and Grzybowski, 2002). The mesoscopic domain is ultimately expected to witness the methodological, technological, and functional convergence of micro/nanosystems engineering with distributed robotic systems (Mastrangeli et al., 2011). Self-assembly in the mesoscopic domain is the object of this perspective.

\section{Mesoscopic Self-Assembly: Past and Present}

Self-assembly so far has been adopted mostly to construct artificial systems. Construction aims to build systems that are structurally stable after assembly, as well as throughout their functional operations. This objective inherently prompts to the thermodynamic control of self-assembly, prototypically represented by crystallization processes. The thermodynamic (i.e., static) framework has indeed been underlying the vast majority of implementations of self-assembly to date (Grzybowski et al., 2009; Mastrangeli et al., 2009; Tørring et al., 2011). Under thermodynamic control, the target structure is designed to univocally coincide with the global minimum of the free energy landscape associated with its building blocks. In absence of local minima, each step toward the target corresponds to a decrease of the system's free energy. Given the negligible mobility of typical mesoscopic building blocks, kinetic energy is injected from an external source to stir the system along its thermodynamic trajectory. The purposeful provision of surrogate high temperature and Brownian motion is temporary, and the final structure withstands its extinction unaffected. The self-assembling system needs to be posed under the effect of potential or force gradients only as long as it takes to be correctly locked into its most stable state. The latter state is path-independent, and can be reached from a variety of initial conditions. The minimal energy state may be degenerate, which improves self-assembly yield. A structure of high designability can be equivalently assembled out of multiple or redundant sets of building blocks (Hogg, 1999). Static self-assembly has been successfully used in pioneering proofs of concepts and applied to the fabrication of mesoscopic systems of technological relevance (Mastrangeli et al., 2009; Leong et al., 2010). Purely stochastic selfassembly approaches, deliberately inspired by chemical reactions and modeled through reaction network formalisms (Mastrangeli et al., 2011; Mermoud et al., 2012; Miyashita et al., 2013), have been fruitfully modulated with varying degrees of determinism, as well. This requires the introduction of programmable force fields, biasing (Chung et al., 2008), predefined templates (Mastrangeli et al., 2009), and conformational constraints (Leong et al., 2010).

More recently, and after an initial sprawl of directions of investigation, research in mesoscopic self-assembly has been manifesting the tendency to converge to a scenario whereby a subset of mature methods, tailored to driving applications, is surrounded by a fertile cloud of explorative forays. Accordingly, the core of mesoscopic static self-assembly eminently includes (1) the fabrication of twoand three-dimensional colloidal crystalline structures with lowdefect densities and long-range order, suitable for, e.g., photonic, sensing, storage, and catalytic purposes (Rycenga et al., 2009; Grzelczak et al., 2010); (2) the self-folding of three-dimensional voxels out of two-dimensional nets, elicited for the fabrication of large sets of homogeneous, specifically functionalized polyhedra embodying chemical vectors, untethered grippers and the smallest patterned artificial particles to date (Leong et al., 2010; Shenoy and Gracias, 2012); and particularly, (3) the electro-mechanical integration of active devices onto pre-processed substrates for the packaging of standalone functional systems (Mastrangeli et al., 2009). As for the latter, fluidic self-assembly (Crane et al., 2013) of chiplets onto carriers templated with trapping sites of complementary geometry possibly represented the first application of selfassembly in industrial manufacturing; and it presently retains a very competitive edge for large-scale electronic packaging thanks to a recent reel-to-reel embodiment with unprecedented throughput (Park et al., 2014). The convergence to the aforementioned scenario arguably results from a selection among the available options - a selection based upon performance and, where applicable, upon the challenges facing the development of laboratory demonstrations into potential staples of industrial practice. The cost-effectiveness of this translation is also undermined by the competing performance improvement and extension of reach of robotic assembly techniques (Gauthier and Régnier, 2011). Capillary self-alignment (Arutinov et al., 2014) represents conversely the exceptional instance of an originally mesoscopic technique, which complements the robotic integration of solid-state devices (of sizes spanning from hundreds of microns to millimeters) onto patterned carrier substrates, thereby enhancing both throughput and accuracy of pick-and-place (Fukushima et al., 2012; Zhou et al., 2014).

\section{The Need for a Shift}

Is the current situation in mesoscopic self-assembly here to stay, or should it rather be considered a trend susceptible of further developments? In arguing in favor of the latter option, we remind that, besides notable exceptions (Fialkowski et al., 2006; Warren et al., 2012), and in spite of the peculiar features of the mesoscopic domain, past and present instances have merely been skimming the surface of the full potential of self-assembly (Lehn, 2002; Warren et al., 2012). Most of the achievements of mesoscopic selfassembly thus far have indeed involved only a limited number of types of homogeneous building blocks (mostly, a single one), a subset of the possible interactions, and exogenous ways to bring the blocks together, typically in close packing or direct contact. Moreover, most synthesized systems are two-dimensional (selfassembled either on planar templates or at fluid/fluid interfaces), are organized into highly ordered lattice-like patterns, and do not really exploit collective interactions other than through redundant number of blocks relative to available target sites, sheer collisions, and other density-related effects. Therefore, even before turning to technological outputs, there is still a lot more to demonstrate, achieve, and fundamentally learn about self-assembly - particularly at the mesoscale, and both inside and outside its static realm. 
On all these fronts, the actualization of self-assembly's foreseeable potential starts by tackling complexity.

\section{Static Complexity: Three-Dimensional Systems}

Steps toward higher complexity may be pursued by shifting the focus of research toward the static self-assembly of threedimensional structures. Protein folding (Dill and MacCallum, 2012) and DNA origami (Tørring et al., 2011) are inspiring examples for this. At mesoscale, three-dimensional building blocks are successfully and deterministically self-assembled through the folding of two-dimensional nets of panels, whose deterministic wrapping, mediated by flexible hinges, extrinsic stresses, or elastocapillary effects, is algorithmically preset by conformational constraints (Shenoy and Gracias, 2012). The resulting structures are nonetheless essentially hollow shells, whose core is more easily occupied by a fluid than by a solid payload. Alternative to constrained folding is the puzzle approach (Cademartiri and Bishop, 2015), whereby the target structure results from the coordination of building blocks upon close contact (Gracias et al., 2000; Zheng et al., 2006; Macias et al., 2013). Here, the elements informing the potential landscape toward the target structure are mainly represented by the building blocks' design - including shape (Cademartiri et al., 2012; Sacanna et al., 2013), surface functionalization, and relative sophistication and functionality with respect to the target structure (Whitesides and Grzybowski, 2002) - and by the parameters ruling their interactions (anisotropy, range, selectivity), both mutual and with the environment. The puzzle approach is, however, liable to the curse of dimensionality, i.e., the combinatorial increase in the number of structures reachable from a single set of initial building blocks versus the number of possible interactions (Cademartiri and Bishop, 2015). Keeping at bay the surge of configurations accessible to a self-assembling system in its potential landscape represents a major, standing reason of caution along this self-assembly strategy. This issue can nonetheless be normalized and faced in several ways. Systems with higher levels of structural and functional sophistication can be produced, for instance, through programmability and reconfigurability. Programmability amount to partitioning the progression of the construction into stages by presetting the sequence of allowed assembly steps. This approach can be practiced by the controlled enablement and selective structural coding of binding events, and subsumes hierarchical self-assembly techniques (Cademartiri and Bishop, 2015). DNA origami are rapidly affirming as the programmable building blocks of choice for selfassembling nanoscopic $2 \mathrm{D}$ and $3 \mathrm{D}$ functional structures, although still suffering from slow kinetics and limited homogeneity at larger scales (Tørring et al., 2011). Reconfigurability consists instead in resetting the morphology of the system (Mattia and Otto, 2015). In static self-assembly, this is only possible by perturbing the potential landscape through changes in boundary conditions (Mao et al., 2002; Mermoud et al., 2012; Mastrangeli et al., 2014).

\section{Dynamic Complexity: Self-Organization}

A much wider realm of possibilities may, furthermore, be approached by shifting from a strictly static self-assembly
TABLE 1 | Representative instances of mesoscopic self-assembly in their static versus dynamic manifestations.

\begin{tabular}{lll}
\hline Type & Static & Dynamic \\
\hline Prototype & Crystals & $\begin{array}{l}\text { Dissipative structures } \\
\text { Cells }\end{array}$ \\
Micrometric & Colloidal crystals & Multistable reconfigurable \\
& Templated ordering of & colloidal arrays and clusters \\
& non-Brownian particles & Autophoretic and tactic \\
Millimetric & Fluidic self-assembly & particles \\
& 1D: railed & Magnetohydrodynamic \\
& 2D: flotation and immersion & self-assembly \\
& forces, capillary self-alignment & \\
& 3D: self-folding, origami & \\
\hline
\end{tabular}

framework to a dynamic one (Table 1) (Warren et al., 2012). Dynamic self-assembly stems from the kinetic control of systems that are thermodynamically metastable or far from equilibrium (Lehn, 2002; Fialkowski et al., 2006; Mattia and Otto, 2015). Metastable systems are kinetically trapped within local minima of the free energy landscape (Mattia and Otto, 2015). The specific self-assembly pathway traversed does select the actual morphology of these systems (Whitelam and Jack, 2015). Seeds (Saitou, 1999) and catalysts (Miyashita et al., 2015) can be devised to displace kinetic barriers and trigger consequent changes in these systems' state. Out of equilibrium or self-organizing systems are instead posed in steady-state by the balance of opposing interactions, such as global attraction and local repulsion or other instances of, respectively, synthetic and dissipative reactions (Mattia and Otto, 2015). A constant energy input, sustaining constant energy dissipation and entropy production, is required to preserve the transient order of such systems, which gets lost upon interruption of the exogenous input. The steady-state actually has a system involved in constant jumps across configurations, which are responsible for the system's collective and emergent properties. Foreshadowed by early demonstrations (Fialkowski et al., 2006; Warren et al., 2012), the hinted shift toward a dynamic framework in mesoscopic self-assembly would once again follow in the steps of supramolecular chemistry, whose evolution of interest is indeed witnessing a drift toward the dynamical paradigm of self-assembly (Mattia and Otto, 2015). In the specific mesoscale case, though, such conceivable move may entail a more radical revision: should the foremost role of self-assembly remain building thermodynamically stable structures, or rather become fostering programmable matter (Warren et al., 2012) through multistable systems? The allure of "going kinetic" further stems from the possibility of ideating structures with self-healing, adaptive, or even emergent properties (Fialkowski et al., 2006; Mattia and Otto, 2015). Analogies with systems chemistry, and elucidation through artificial replication of mechanisms possibly at the origin of life (Nicolis and Prigogine, 1977; Schneider and Kay, 1994; Pross, 2011), are also driving motives. It may be inspiring also to remind that energy dissipation does play a fundamental role in the physics of computation (Bennett, 2003); and, more pragmatically, that all active devices of daily use absolve their functions only when transiently posed out of thermodynamic equilibrium through an external supply of energy, ultimately dissipated into heat. A standing key challenge 
remains devising efficient ways to implement and tune energy input and dissipation in mesoscopic self-organizing systems. In this respect, progress is nevertheless being made. Colloidal systems are witnessing a rapid increase of demonstrations of selforganization and dynamic self-assembly (Snezhko, 2011; Kokot et al., 2015); and the upper end of the mesoscopic domain is starting to sport a collection of remarkable instances, such as those staged through variations of a magnetohydrodynamic setup (Fialkowski et al., 2006), by variably coordinating sets of selfpropelled particles of diverse nature (Ismagilov et al., 2002; Soh et al., 2008, 2011), and even through mechanical vibration of inert granulates (Marchetti et al., 2013). Even by constraining selfassembling systems to one- or two-dimensional playgrounds like microfluidic channels and fluid/fluid or fluid/solid interfaces, respectively - the conjunct effect of multiple, superposed, exogenous potential or force gradients and of autonomous locomotion of autophoretic particles may usher into unprecedented systems

\section{References}

Arutinov, G., Smits, E. C., Albert, P., Lambert, P., and Mastrangeli, M. (2014). Inplane mode dynamics of capillary self-alignment. Langmuir 30, 13092-13102. doi:10.1021/la502831r

Ball, P. (2011). Shapes: Nature's Patterns: A Tapestry in Three Parts. Oxford: Oxford University Press.

Bennett, C. H. (2003). Notes on Landauer's principle, reversible computation, and Maxwell's Demon. Stud. Hist. Philos. Sci. B Stud. Hist. Philos. Mod. Phys. 34, 501-510. doi:10.1016/S1355-2198(03)00039-X

Bishop, K. J., Wilmer, C. E., Soh, S., and Grzybowski, B. A. (2009). Nanoscale forces and their uses in self-assembly. Small 5, 1600-1630. doi:10.1002/smll.200900358

Cademartiri, L., and Bishop, K. J. (2015). Programmable self-assembly. Nat. Mater. 14, 2-9. doi:10.1038/nmat4184

Cademartiri, L., Bishop, K. J., Snyder, P. W., and Ozin, G. A. (2012). Using shape for self-assembly. Philos. Trans. A Math. Phys. Eng. Sci. 370, 2824-2847. doi:10. 1098/rsta.2011.0254

Chung, S. E., Park, W., Shin, S., Lee, S. A., and Kwon, S. (2008). Guided and fluidic self-assembly of microstructures using railed microfluidic channels. Nat. Mater. 7, 581-587. doi:10.1038/nmat2208

Crane, N. B., Onen, O., Carballo, J., Ni, Q., and Guldiken, R. (2013). Fluidic assembly at the microscale: progress and prospects. Microfluid. Nanofluidics 14, 383-419. doi:10.1007/s10404-012-1060-1

Dill, K. A., and MacCallum, J. L. (2012). The protein-folding problem, 50 years on. Science 338, 1042-1046. doi:10.1126/science.1219021

Fialkowski, M., Bishop, K. J., Klajn, R., Smoukov, S. K., Campbell, C. J., and Grzybowski, B. A. (2006). Principles and implementations of dissipative (dynamic) self-assembly. J. Phys. Chem. B 110, 2482-2496. doi:10.1021/jp054153q

Fukushima, T., Iwata, E., Ohara, Y., Murugesan, M., Bea, J., Lee, K., et al. (2012). Multichip-to-wafer three-dimensional integration technology using chip selfassembly with excimer lamp irradiation. IEEE Trans. Electron. Devices 59, 2956-2963. doi:10.1109/TED.2012.2212709

Gauthier, M., and Régnier, S. (2011). Robotic Micro-Assembly. Hoboken, NJ: John Wiley \& Sons.

Gracias, D. H., Tien, J., Breen, T. L., Hsu, C., and Whitesides, G. M. (2000). Forming electrical networks in three dimensions by self-assembly. Science 289, 1170-1172. doi:10.1126/science.289.5482.1170

Groß, R., and Dorigo, M. (2008). Self-assembly at the macroscopic scale. Proc. IEEE 96, 1490-1508. doi:10.1109/JPROC.2008.927352

Grzelczak, M., Vermant, J., Furst, E. M., and Liz-Marzán, L. M. (2010). Directed self-assembly of nanoparticles. ACS Nano 4, 3591-3605. doi:10.1021/nn100869j

Grzybowski, B. A., Wilmer, C. E., Kim, J., Browne, K. P., and Bishop, K. J. (2009). Self-assembly: from crystals to cells. Soft Matter 5, 1110-1128. doi:10.1039/ b902046m

Hogg, T. (1999). Robust self-assembly using highly designable structures. Nanotechnology 10, 300. doi:10.1088/0957-4484/10/3/313 with innovative properties. Along the way, this may provide important additional hints toward a comprehensive theory of dissipative systems (Nicolis and Prigogine, 1977; Tretiakov et al., 2009a,b, 2013) and, eventually, of living systems (Schneider and Kay, 1994; Mann, 2008).

\section{Complexity is the Key}

While not dismissing the persistent development of alternative approaches able to leverage or complement current manufacturing practices, self-assembly should ultimately concentrate on its unicity and target achievements that are exclusively accessible to itself. This will let self-assembly thrive securely guarded from applicative competition. Complexity - declined through collective interactions of large sets of heterogeneous components, structures with fully three-dimensional architectures, and kinetically controlled systems - holds the promise to light up the road ahead.

Ismagilov, R. F., Schwartz, A., Bowden, N., and Whitesides, G. M. (2002) Autonomous movement and self-assembly. Angew. Chem. Int. Ed. Engl. 114, 674-676. doi:10.1002/1521-3757(20020215)114:4<674::AID-ANGE674> 3.0.CO; $2-\mathrm{Z}$

Israelachvili, J. N. (2011). Intermolecular and Surface Forces: Revised Third Edition. Oxford: Academic press.

Kokot, G., Piet, D., Whitesides, G. M., Aranson, I. S., and Snezhko, A. (2015). Emergence of reconfigurable wires and spinners via dynamic self-assembly. Sci. Rep. 5, 9528. doi:10.1038/srep09528

Lehn, J.-M. (2002). Toward complex matter: supramolecular chemistry and selforganization. Proc. Natl. Acad. Sci.U.S.A. 99, 4763-4768. doi:10.1073/pnas. 072065599

Leong, T. G., Zarafshar, A. M., and Gracias, D. H. (2010). Three-dimensional fabrication at small size scales. Small 6, 792-806. doi:10.1002/smll.200901704

Macias, N., Pandey, S., Deswandikar, A., Kothapalli, C., Yoon, C., Gracias, D., et al. (2013). "A cellular architecture for self-assembled 3D computational devices," in IEEE/ACM International Symposium on, Nanoscale Architectures (NANOARCH) (New York City, NY: IEEE).

Mann, S. (2008). Life as a nanoscale phenomenon. Angew. Chem. Int. Ed. 47, 5306-5320. doi:10.1002/anie.200705538

Mao, C., Thalladi, V. R., Wolfe, D. B., Whitesides, S., and Whitesides, G. M. (2002). Dissections: self-assembled aggregates that spontaneously reconfigure their structures when their environment changes. J. Am. Chem. Soc. 124, 14508-14509. doi:10.1021/ja021043d

Marchetti, M., Joanny, J., Ramaswamy, S., Liverpool, T., Prost, J., Rao, M., et al. (2013). Hydrodynamics of soft active matter. Rev. Mod. Phys. 85, 1143. doi:10. 1103/RevModPhys.85.1143

Mastrangeli, M., Abbasi, S., Varel, C., Van Hoof, C., Celis, J.-P., and Böhringer, K. F. (2009). Self-assembly from milli-to nanoscales: methods and applications. J. Micromech. Microeng. 19, 083001. doi:10.1088/0960-1317/19/8/083001

Mastrangeli, M., Mermoud, G., and Martinoli, A. (2011). Modeling self-assembly across scales: the unifying perspective of smart minimal particles. Micromachines 2, 82-115. doi:10.3390/mi2020082

Mastrangeli, M., Schill, F., Goldowsky, J., Knapp, H., Brugger, J., and Martinoli, A. (2014). "Automated real-time control of fluidic self-assembly of microparticles," in IEEE International Conference on, Robotics and Automation (ICRA) (Hong Kong, CN: IEEE).

Mattia, E., and Otto, S. (2015). Supramolecular systems chemistry. Nat. Nanotechnol. 10, 111-119. doi:10.1038/nnano.2014.337

Mermoud, G., Mastrangeli, M., Upadhyay, U., and Martinoli, A. (2012). "Realtime automated modeling and control of self-assembling systems," in IEEE International Conference on, Robotics and Automation (ICRA) (Saint Paul, MN: IEEE).

Miyashita, S., Audretsch, C., Nagy, Z., Füchslin, R. M., and Pfeifer, R. (2015). Mechanical catalysis on the centimetre scale. J. R. Soc. Interface 12, 20141271. doi:10.1098/rsif.2014.1271 
Miyashita, S., Diller, E., and Sitti, M. (2013). Two-dimensional magnetic micromodule reconfigurations based on inter-modular interactions. Int. J. Rob. Res. 32, 591-613. doi:10.1177/0278364913479837

Nicolis, G., and Prigogine, I. (1977). Self-Organization in Nonequilibrium Systems. New York, NY: Wiley.

Park, S. C., Fang, J., Biswas, S., Mozafari, M., Stauden, T., and Jacobs, H. O. (2014). A first implementation of an automated reel-to-reel fluidic self-assembly machine. Adv. Mater. Weinheim 26, 5942-5949. doi:10.1002/adma.201401573

Pross, A. (2011). Toward a general theory of evolution: extending Darwinian theory to inanimate matter. J. Syst. Chem. 2, 1-1. doi:10.1186/1759-2208-2-1

Rycenga, M., Camargo, P. H., and Xia, Y. (2009). Template-assisted self-assembly: a versatile approach to complex micro-and nanostructures. Soft Matter 5, 1129-1136. doi:10.1039/B811021B

Sacanna, S., Korpics, M., Rodriguez, K., Colón-Meléndez, L., Kim, S.-H., Pine, D. J., et al. (2013). Shaping colloids for self-assembly. Nat. Commun. 4, 1688. doi: $10.1038 /$ ncomms 2694

Saitou, K. (1999). Conformational switching in self-assembling mechanical systems. IEEE Trans. Rob. Autom. 15, 510-520. doi:10.1109/70.768183

Schneider, E. D., and Kay, J. J. (1994). Life as a manifestation of the second law of thermodynamics. Math. Comput. Model. 19, 25-48. doi:10.1016/0895-7177(94) 90188-0

Shenoy, V. B., and Gracias, D. H. (2012). Self-folding thin-film materials: from nanopolyhedra to graphene origami. MRS Bull. 37, 847-854. doi:10.1557/mrs. 2012.184

Snezhko, A. (2011). Non-equilibrium magnetic colloidal dispersions at liquid-air interfaces: dynamic patterns, magnetic order and self-assembled swimmers. J. Phys. Condens. Matter 23, 153101. doi:10.1088/0953-8984/23/15/153101

Soh, S., Bishop, K. J., and Grzybowski, B. A. (2008). Dynamic self-assembly in ensembles of camphor boats. J. Phys. Chem. B 112, 10848-10853. doi:10.1021/ jp7111457

Soh, S., Branicki, M., and Grzybowski, B. A. (2011). Swarming in shallow waters. J. Phys. Chem. Lett. 2, 770-774. doi:10.1021/jz200180z

Tørring, T., Voigt, N. V., Nangreave, J., Yan, H., and Gothelf, K. V. (2011). DNA origami: a quantum leap for self-assembly of complex structures. Chem. Soc. Rev. 40, 5636-5646. doi:10.1039/c1cs15057j

Tretiakov, K. V., Bishop, K. J., and Grzybowski, B. A. (2009a). Additivity of the excess energy dissipation rate in a dynamically self-assembled system. J. Phys. Chem. B 113, 7574-7578. doi:10.1021/jp811473q
Tretiakov, K. V., Bishop, K. J., and Grzybowski, B. A. (2009b). The dependence between forces and dissipation rates mediating dynamic self-assembly. Soft Matter 5, 1279-1284. doi:10.1039/b811254a

Tretiakov, K. V., Szleifer, I., and Grzybowski, B. A. (2013). The rate of energy dissipation determines probabilities of non-equilibrium assemblies. Angew. Chem. Int. Ed. Engl. 125, 10494-10498. doi:10.1002/ange.201301386

Vicsek, T., and Zafeiris, A. (2012). Collective motion. Phys. Rep. 517, 71-140. doi:10.1016/j.physrep.2012.03.004

Warren, S. C., Guney-Altay, O., and Grzybowski, B. A. (2012). Responsive and nonequilibrium nanomaterials. J. Phys. Chem. Lett. 3, 2103-2111. doi:10.1007/ s00249-012-0815-7

Whitelam, S., and Jack, R. L. (2015). The statistical mechanics of dynamic pathways to self-assembly. Annu. Rev. Phys. Chem. 66, 143-163. doi:10.1146/ annurev-physchem-040214-121215

Whitesides, G. M., and Boncheva, M. (2002). Beyond molecules: self-assembly of mesoscopic and macroscopic components. Proc. Natl. Acad. Sci. U.S.A. 99, 4769-4774. doi:10.1073/pnas.082065899

Whitesides, G. M., and Grzybowski, B. (2002). Self-assembly at all scales. Science 295, 2418-2421. doi:10.1126/science.1070821

Whitesides, G. M., Mathias, J. P., and Seto, C. T. (1991). Molecular self-assembly and nanochemistry: a chemical strategy for the synthesis of nanostructures. Science 254, 1312-1319. doi:10.1126/science.1962191

Zheng, W., Chung, J., and Jacobs, H. O. (2006). Fluidic heterogeneous microsystems assembly and packaging. J. Microelectromech. Syst. 15, 864-870. doi:10.1109/ JMEMS.2006.878885

Zhou, Q., Liimatainen, V., and Routa, I. (2014). "Microsystem integration using hybrid microassembly," in Electronics System-Integration Technology Conference (ESTC) (Helsinki, FI: IEEE).

Conflict of Interest Statement: The author declares that the research was conducted in the absence of any commercial or financial relationships that could be construed as a potential conflict of interest.

Copyright (C) 2015 Mastrangeli. This is an open-access article distributed under the terms of the Creative Commons Attribution License (CC BY). The use, distribution or reproduction in other forums is permitted, provided the original author(s) or licensor are credited and that the original publication in this journal is cited, in accordance with accepted academic practice. No use, distribution or reproduction is permitted which does not comply with these terms. 